De nouvelles formes de gentrification?

Dynamiques résidentielles et commerciales à Château-Rouge (Paris)

\title{
Marie Chabrol
}

\section{CpenEdition}

Journals

Édition électronique

URL : http://journals.openedition.org/cdg/1031

DOI : $10.4000 /$ cdg. 1031

ISSN : 2107-7266

Éditeur

UMR 245 - CESSMA

Référence électronique

Marie Chabrol, « De nouvelles formes de gentrification ? Dynamiques résidentielles et commerciales à Château-Rouge (Paris) », Carnets de géographes [En ligne], 4 | 2012, mis en ligne le 01 septembre 2012, consulté le 24 septembre 2020. URL : http://journals.openedition.org/cdg/1031 ; DOI : https://doi.org/ $10.4000 /$ cdg. 1031

\section{(c) $($ ) $(9)$}

La revue Carnets de géographes est mise à disposition selon les termes de la Licence Creative Commons Attribution - Pas d'Utilisation Commerciale - Pas de Modification 4.0 International. 


\title{
De nouvelles formes de gentrification? Dynamiques résidentielles et commerciales à Château-Rouge (Paris)
}

\author{
MARIE CHABROL
}

Cette thèse a pour objet d'étude les dimensions physiques et sociales d'une situation de recomposition urbaine, dans un ancien quartier populaire et très commerçant. Elle montre la coexistence durable de deux dynamiques autonomes, résidentielles et commerciales, l'une contribuant à l'avancement du processus de gentrification sur le plan résidentiel tandis que l'autre consolide la spécialisation d'un pôle de commerces africains.

Château-Rouge est la partie centrale de la Goutte d'Or, l'un des espaces les plus populaires du nord de Paris, transformé depuis le début des années 2000 par l'installation de populations plus aisées que les habitants déjà en place et par des actions publiques portant sur l'habitat, les espaces publics et les activités commerciales. Ce quartier compte près de 300 commerces, dont près des deux tiers sont tournés vers une clientèle d'origine africaine, qui ne réside pas sur place, mais qui contribue à donner de cet espace une image de quartier africain. Dans ce contexte de changement de la composition sociale de la population des résidents et de reprise en main du quartier par les pouvoirs publics, le maintien des usages liés aux commerces amène à poser de nouvelles questions sur le déroulement du processus de gentrification, plus complexe et moins linéaire que décrit dans la littérature.

Avec une double entrée par les temporalités urbaines et par les pratiques de mobilité, l'objectif de ce travail est de comprendre une situation complexe, de montrer comment, mais aussi pourquoi, peuvent coexister des temporalités différentes (notamment entre changement résidentiel et changement commercial), comment peuvent perdurer des situations de côtoiement de populations socialement diversifiées sur un temps long, et quelles nouvelles formes urbaines peuvent engendrer ces coexistences.

Ces positionnements théoriques et le choix d'une démarche très locale ont nécessité la mise en place d'un système d'investigation articulant différents modes de collecte de données sur la constitution du quartier autant que sur son fonctionnement actuel. En plus du rassemblement de données déjà existantes et d'une pratique du terrain continue, deux enquêtes par questionnaires ont été menées. La première, qui concerne cinq immeubles choisis en fonction de leur type de bâti, avait pour objectif d'éclairer les évolutions du quartier de l'intérieur et de relier les mobilités résidentielles des individus à l'évolution du cadre physique et du peuplement des immeubles. De l'information directe a ainsi été recueillie sur 60 personnes (leur trajectoire, résidentielle et personnelle, collectée grâce à des matrices biographiques, mais aussi leurs pratiques quotidiennes et leur perception du quartier). Des données ont aussi été recueillies sur plus de 70 logements, dont la trajectoire a pu être reconstituée pour certains depuis le milieu du $\mathrm{XX}^{\mathrm{e}}$ siècle. La seconde enquête a été menée dans les rues commerçantes de Château-Rouge. Elle visait l'ensemble des usagers de cet espace, avec pour objectif d'éclairer le fonctionnement de la centralité africaine, mais aussi de savoir comment elle était vécue et perçue. 600 questionnaires ont été renseignés lors de deux 
journées d'enquête, complétant un ensemble de données déjà obtenues par l'observation et par des entretiens avec les commerçants.

La première partie de résultats de la thèse met en évidence les structures et les acteurs de l'offre résidentielle et commerciale du quartier. Bien que très hétérogène, le cadre bâti a été conçu au XIX ${ }^{\mathrm{e}}$ pour loger des populations d'ouvriers et d'employés. Très peu retouché au cours du $\mathrm{XX}^{\mathrm{e}}$ siècle, ce cadre spatial a évolué de manière différenciée en fonction des secteurs du quartier et est entré dans un cycle de dévalorisation à partir des années 1980. À la fin des années 1990, une intervention publique et la découverte d'un parc de logements anciens, au moment où les prix de l'immobilier ont augmenté dans la capitale, ont eu pour conséquence de faire démarrer un processus de gentrification du quartier.

La seconde partie de résultats, plus directement issue des deux enquêtes, décrypte le fonctionnement actuel de cet espace, et montre qu'au fil des ans, loin de réduire les différences entre les espaces résidentiels, l'avancement du processus de gentrification semble au contraire aboutir à un morcellement sur le plan spatial. Il progresse et se consolide dans certains secteurs (les étages en hauteur, les immeubles en pierre de taille), mais ne touche pas les autres. Des micro-secteurs apparaissent ainsi à différentes échelles et constituent une mosaïque contrastée, où coexistent des situations de changement, en voie de consolidation, et des continuités.

Cette mosaïque spatiale et sociale est fonctionnellement renforcée par deux phénomènes liés l'un à l'autre : le maintien de la centralité commerciale africaine et l'adaptation des pratiques des résidents. Plus de trente ans après l'ouverture des premières boutiques, et malgré la reprise en main de cet espace par les pouvoirs publics, la centralité africaine de ChâteauRouge demeure très dynamique. Elle continue d'attirer une clientèle venue des quartiers voisins $\mathrm{du} 18^{\mathrm{e}}$ arrondissement, du reste de Paris, de toute l'Île-de-France, de province et même de l'étranger, et qui, par ses pratiques très diverses, contribue au maintien et au renouvellement de l'offre commerciale. Regroupés, diversifiés et bon marché, ces commerces sont fréquentés par différents types d'usagers et, comme d'autres centralités minoritaires, cet espace assume aussi des fonctions sociales importantes qui contribuent elles aussi au renouvellement permanent de l'image africaine de ce lieu.

Le fonctionnement de cette centralité commerciale n'est pas un frein à la gentrification, mais n'est pas pour autant sans effet sur les pratiques des résidents, notamment les pratiques d'approvisionnement. La plupart des résidents ont un usage faible des commerces du quartier, qui ne répondent pas à leurs besoins et prend en compte une échelle plus large d'offre commerciale, notamment dans les quartiers proches, renforçant ainsi les dynamiques commerciales de ces espaces-là.

$\mathrm{Au}$ total, deux dynamiques autonomes, résidentielles et commerciales, se consolident à l'échelle locale, chacune étant alimentée par des processus s'inscrivant dans des logiques qui se situent à l'extérieur du quartier. En conséquence, il semble bien qu'il ne s'agisse pas ici d'une situation de transition, mais bien d'une configuration aboutie de changement social, qui amène à remettre en question le modèle linéaire et aréolaire de la gentrification. Ces résultats enrichissent les connaissances sur le déroulement du processus de gentrification dans le contexte de quartiers anciens marqués par des activités commerciales liées à l'immigration, comme il en existe d'autres à Paris et dans d'autres métropoles. Ils contribuent aussi à poser de nouvelles questions sur le maintien de pratiques populaires dans des métropoles décrites comme toujours plus embourgeoisées. 
Fiche informative

\section{Lien électronique pour télécharger la thèse}

http://tel.archives-ouvertes.fr/tel-00658852/fr/

\section{Discipline}

Géographie

\section{Directrice}

Françoise DUREAU (DR IRD)

\section{Université}

Université de Poitiers

Membres du jury de thèse, soutenue le 29 novembre 2011 :

Brigitte Bertoncello, Professeur, Université de Provence (examinatrice)

Françoise Dureau, Directrice de recherche, IRD (directrice de la thèse)

Yankel Fijalkow, Professeur, ENSA Paris Val-de-Seine (rapporteur)

Jean-Pierre Lévy, Directeur de recherche, CNRS (président du jury)

Emmanuel Ma Mung, Directeur de recherche, CNRS (examinateur)

Thérèse Saint-Julien, Professeur émérite, Université Paris I (rapporteur)

\section{Situation actuelle}

ATER Université Paris-Sorbonne / membre du laboratoire Migrinter - UMR 6588 CNRS / Université de Poitiers

\section{Contact}

machabrol@gmail.com 